\title{
FELIU ARQUIOLA, ELENA (ED.) (2018). PROBLEMAS DE DEMARCACIÓN EN MORFOLOGÍA Y SINTAXIS DEL ESPAÑOL. DEMARCATION PROBLEMS IN MORPHOLOGY AND SPANISH SINTAX. PETER LANG, SERIE FONDO HISPÁNICO DE LINGÜÍSTICA Y FILOLOGÍA. 198 PÁGINAS
}

\author{
Abigail CARretero \\ Universidad Autónoma de Querétaro (México) \\ abbie.carretero@fulbrightmail.org \\ https://orcid.org/0000-0002-8416-885X
}

La demarcación categorial de ciertas unidades lingüísticas ha sido una cuestión compleja que ha mantenido la atención de los estudiosos de la gramática del español. Este libro propone un acercamiento selectivo a nueve cuestiones particularmente controvertidas, a las que se dedican sendos capítulos. Temas tales como los adverbios en -mente, los adverbios no concordados o los cuantificadores nominales no prototípicos son, entre otros, elementos de difícil adscripción de acuerdo con los patrones gramaticales al uso, por lo que no es de extrañar que hayan sido objeto de un prolongado debate. La monografía editada por Elena Felíu, en la que participan investigadores de las universidades españolas de Jaén y Málaga, ofrece al respecto aportaciones teóricas y descriptivas que indudablemente enriquecen tal controversia desde una óptica contemporánea. Los nueve capítulos se agrupan en tres partes, dedicadas respectivamente a cuestiones intramorfológicas, a la relación entre morfología y sintaxis y, finalmente, a los límites entre determinadas construcciones sintácticas. A eso se suman un capítulo introductorio, que expone brevemente los objetivos y estructura del libro, y unas breves notas biobibliográficas de los autores como cierre.

Francisco Carriscondo Esquivel firma los tres primeros capítulos del libro, que se agrupan en la sección preliminar. El trabajo inaugural versa sobre los adverbios en -mente. El autor muestra inicialmente la descripción del fenómeno de acuerdo a lo expuesto por los antecedentes bibliográficos, para después afrontar su análisis en cuatro apartados, en virtud de las posibles hipótesis interpretativas. El primero consideraría las formaciones en -mente como formas de flexión del adjetivo desde el punto de vista del caso. En segundo lugar, se contempla la hipótesis de la composición adverbial (compuesto léxico). Posteriormente se analiza la vía de composición sintagmática / sintáctica (compuestos frasales) para, finalmente, atender la posibilidad de la derivación. Carriscondo reconoce la falta de consenso a

Para citar esta reseña / To cite this book review: Carretero, Abigail (2020). Reseña de Elena Feliu Arquiola (ed.) (2018). Problemas de demarcación en morfología y sintaxis del español. Demarcation problems in morphology and spanish sintax. Peter Lang, Serie Fondo Hispánico de Lingüística y Filología. 198 p., 2018. ELUA, 34: 221-225. https://doi.org/10.14198/ELUA2020.34.11

Enlace / Link: https://doi.org/10.14198/ELUA2020.34.11

(c) BY-NC Este trabajo se publica bajo una licencia de Creative Commons Reconocimiento-NoComercial 4.0 Internacional 
propósito de estas formaciones, como queda de relieve incluso en la más reciente gramática académica. Si bien admite que la posibilidad de la derivación va ganando adeptos en fechas recientes, él considera que es el análisis fundado en la composición léxica el que se ajusta más a las peculiaridades gramaticales que estas unidades conservan en la sincronía actual.

El segundo trabajo se dedica a los sustantivos participiales, poniendo el foco en el tratamiento que les otorgan actualmente la Real Academia Española y la Asociación de Academias de la Lengua Española. El autor parte de la asunción de que, dentro de la etiqueta general de "derivados participiales" (partido, amanecida, etc.) conviven cinco esquemas morfológicos de naturaleza diferente, por más que resulten cuasihomógrafos: el primero [a/i] - $d$-o/a; el segundo -a/id-o/a; el tercero -ado; el cuarto - ada; y, el quinto -ido. A su vez, los dos modelos iniciales cuentan con subdivisiones específicas en virtud de sus posibilidades de categorización. Ello se debe a que, si bien los cinco esquemas generan derivados nominales, solo los dos primeros admiten derivados adjetivales y únicamente el primero ofrece formas puramente participiales de naturaleza verbal. A partir de ahí, Carriscondo examina la atención que reciben estas unidades en las dos principales obras académicas de referencia en la actualidad: la Nueva Gramática de la Lengua Española (2009) y el Diccionario de la Lengua Española (2014). Dicho análisis resulta muy revelador porque pone de relieve la existencia de notables discrepancias entre ambas y, en general, un tratamiento poco consistente. A su juicio, eso se debe a que las instituciones académicas han optado prioritariamente por un criterio semántico, mientras que este autor considera que los criterios morfológico e histórico posibilitan un análisis mucho más acorde con los datos.

La última aportación de Francisco Carriscondo Esquivel lleva por título "Cuestiones morfológicas menores que afectan al nivel fónico-gráfico". De nuevo, el autor centra su interés en algunas cuestiones problemáticas detectadas en las obras académicas. En este caso, aludirá a dos versiones de la gramática (la Maior de 2009 y la Manual de 2010) y a la Ortografía de la lengua española (2010). Son tres las cuestiones que se toman aquí en consideración. Cabe dividir el trabajo en dos partes: la primera dedicada a cuestiones morfo-fonológicas (la distinción entre morfema, infijo e interfijo y, como corolario, la categorización de la vocal temática de los verbos); la segunda, al uso del guion, un elemento ortográfico aparentemente marginal pero que, como se enfatiza en estas páginas, tiene implicaciones morfológicas relevantes particularmente para el análisis de las formas compuestas. Carriscondo constata la aparente contradicción de algunas definiciones académicas en materia morfológica. Si se admite que el morfema es la unidad mínima dotada de significado, cabe admitir como tal al infijo, en tanto que elemento gramatical insertado en el interior de la palabra con contenido semántico propio; sí puede ser calificado como morfema. En cambio, eso no sería aplicable al interfijo. Resulta contradictorio, por tanto, definirlo como un morfema carente de significado. De acuerdo con ello, el autor estima que la vocal temática de los verbos es un interfijo y, por tanto, no es realmente un morfema. Se trata de un elemento semánticamente vacío cuya presencia en el verbo viene motivada por causas estrictamente morfológicas. Por lo que se refiere al uso del guion, se resalta el papel que la Academia le atribuye como signo de unión entre palabras o unidades gramaticales, particularmente en el caso de los llamados "compuestos univerbales". Lo que ocurre es que aquí también se detectan ciertas inadecuaciones en la doctrina académica, especialmente llamativas en los compuestos adjetivales en los que el primer elemento empieza a asimilarse a un prefijo (¿se ha de escribir superficie cóncavo-convexa o superficie cóncavoconvexa?). 
La segunda parte del libro cuenta con tres trabajos que describen la demarcación entre morfología y sintaxis, al igual que los límites entre clases de palabras. El trabajo inicial, de Elena Felíu Arquiola, trata sobre las dificultades que entraña el análisis de los prefijos separables, del tipo: ejercicios pre y post parto. El primero de los problemas concierne a su estatuto gramatical (prefijos, preposiciones o unidades mixtas); el segundo se basa en la naturaleza categorial de las palabras prefijadas resultantes cuando modifican específicamente a sustantivos (manifestación proaborto, tarjeta prepago, etc.); finalmente, el tercero nace de las estructuras de coordinación (pro y antisamperistas), en la medida en que podrían considerarse análogas a la coordinación de preposiciones (por y para la venganza). La autora concluye que es posible que el banco de prefijos prototípicos en español sea más reducido que lo que se cree habitualmente, y que muchas unidades calificadas como tales requieran una catalogación diferente, ya sea como elementos compositivos, prefijoides o alguna otra.

En el segundo trabajo Felíu llama la atención sobre la posible existencia de adverbios concordados en español, es decir, dotados de variación morfológica de género y número. La autora señala (p. 105) que bajo esta etiqueta cabe reconocer al menos tres fenómenos distintos. Uno de ellos sería la modificación de verbos (María habla rápida), que la autora no toma en consideración y que, aunque no se indique explícitamente, genera dudas en cuanto al carácter adverbial o adjetival del modificador, al tratarse de posibles casos de predicación secundaria o "complemento predicativo". Un segundo caso vendría dado por la flexión de adverbios focalizadores (solos tres), y un tercero, que es el que ocupa realmente este capítulo, por los adverbios que intervienen en la modificación adjetival (medios perezosos). En relación con este último, Felíu también constata ejemplos de adverbios concordados cuando modifican a otros adverbios (ella está media mal). Generalmente, la bibliografía previa sitúa estos casos especiales en variedades no estándares del español de América. No obstante, también está atestiguada su presencia en el español europeo, amén de ser un fenómeno que cuenta con paralelismos en otras lenguas romances. Por tanto, la autora aboga por aceptar que, al menos potencialmente, los rasgos flexivos del sintagma nominal pueden ser irradiados al adverbio si la estructura morfológica de este último así lo permite.

La segunda parte del volumen se cierra con la contribución de Ventura Salazar García, dedicada a lo que él denomina cuantificadores nominales no prototípicos, que son aquellos en los que el significado cuantitativo coexiste con un contenido denotativo. Tras revisar la bibliografía previa y constatar los problemas de categorización inherentes a estas unidades, el trabajo lleva a cabo una serie de precisiones terminológicas, destinadas a justificar la denominación finalmente elegida. Posteriormente, lleva a cabo una clasificación de estos cuantificadores a partir de un criterio semántico. Ello lo lleva a reconocer dos subcategorías básicas. La primera vendría dada por los nombres acotadores (loncha, lingote, tableta, etc.) y la segunda por los cuantificadores analógicos (botella, vaso, dedo, montón, etc.). Dentro de esta última cabría diferenciar a su vez entre la cuantificación mensurativa y la evaluativa. La parte final del capítulo está dedicada al papel que desempeñan estos cuantificadores en la distinción entre construcciones partitivas y pseudopartitivas. El autor critica el tratamiento que se da a esta cuestión desde postulados formalistas, que son los que subyacen en la Nueva Gramática de la Lengua Española (2009), donde se plantea una relación biunívoca entre cuantificación nominal y construcción pseudopartitiva. Como alternativa, plantea un análisis de orientación funcional donde la diferencia entre la construcción partitiva y la pseudopartitiva descansa en sus condiciones de referencia y no en la categorización sintáctica del cuantificador. 
La tercera parte del libro atiende los límites entre construcciones sintácticas. Corre a cargo de Carmen Conti Jiménez, que es la autora de los tres capítulos que lo componen. El primero de ellos trata sobre las cláusulas adverbiales. La autora inicia su análisis con una descripción bien documentada de las clasificaciones precedentes que las cláusulas adverbiales han recibido en distintos trabajos previos, tanto en términos semánticos como sintácticos. Todo ello queda sintetizado en cinco tablas (pp. 158 y s.) en las que se toman en consideración las cláusulas temporales, modales, espaciales, finales, causales, condicionales, concesivas, comparativas, consecutivas ponderativas e ilativas. Finalmente, la autora pone de relieve que todas esas propuestas clasificatorias pueden subdividirse en cuatro grandes grupos: propuestas funcionales, clasificaciones basadas en la segmentación del nexo, las que exceden los límites de la subordinación y las basadas en el nivel de adjunción.

El segundo trabajo de Conti se dedica a los dos tipos de construcciones consecutivas que más problemas clasificatorios suscitan: las ponderativas o escalares, donde el primer miembro de la estructura debe contar con un intensificador (tal, tan, tantos), y las ilativas, que tienen un claro sentido conclusivo y se marcan mediante nexos no correlativos (luego, conque, de modo que, etc.). La autora contempla las diversas adscripciones de que han sido objeto tales estructuras, constatando su gran variabilidad. Las ponderativas han sido consideradas, bien subordinadas adverbiales, bien una variante de las oraciones de relativo, mientras que en fechas recientes han sido interpretadas como adyacentes del intensificador o del sintagma intensificado. Por su parte, las ilativas se han clasificado, según los autores, entre las subordinadas, las yuxtapuestas o las coordinadas. Aunque Carmen Conti no llega a desarrollar por extenso una propuesta propia, sus argumentos abogan claramente por la idea de que las consecutivas ilativas constituyen un caso de coordinación, y las ponderativas de subordinación adverbial, toda vez que los datos apuntan a que la segunda cláusula depende sintáctica y semánticamente de la primera, y a la vez es un constituyente estructural y funcionalmente separable de la partícula intensificadora.

Para cerrar la tercera sección del libro, Conti Jiménez ofrece un breve capítulo acerca de si las cláusulas adversativas deben clasificarse entre las oraciones coordinadas o entre las interordinadas. Aunque tradicionalmente se asumía que constituían ejemplos claros de coordinación, el hecho de que muestren una relación semántica compleja y que siempre aparezcan en estructuras binarias ha llevado a algunos autores a plantearlas como casos de una nueva relación interclausal, conocida como interordinación o interdependencia. A partir de ahí, la autora somete estas oraciones a diversas pruebas sintácticas que le permiten concluir que las cláusulas adversativas con pero cumplen todas las propiedades atribuibles en español a las oraciones coordinadas. En cambio, las cláusulas con aunque y con sino se alejan de ese prototipo y muestran evidentes similitudes con las cláusulas subordinadas, lo que permite postular en ellas cierto grado de hibridación.

Como conclusión, debo ponderar el rigor y la claridad que caracterizan todos los trabajos reunidos en este volumen, que sin duda está llamado a ocupar un lugar significativo en la bibliografía contemporánea de la investigación gramatical del español. Todos ellos arrojan nueva luz, tanto en el plano metodológico como en el descriptivo, sobre cuestiones sumamente intrincadas que se han prestado a la discrepancia desde antiguo. No obstante, también me siento obligada a advertir que los resultados de cada colaboración distan de ser homogéneos. Particularmente algunas de las firmadas por Carriscondo y por Felíu parecen excesivamente escuetas; plantean adecuadamente el problema y ofrecen un buen diagnósti- 
co, pero les falta llevar su línea argumentativa hasta el final, con la presentación y defensa de una hipótesis propia. En el otro extremo cabría situar el bien documentado capítulo de Carmen Conti acerca de las cláusulas adverbiales y, en especial, el de Ventura Salazar García sobre la cuantificación nominal. Se trata de única aportación de este autor, pero, a cambio, es con diferencia la más extensa y, asimismo, la que asume un mayor grado de compromiso teórico. Por ende, encaja de manera más precisa con lo que se espera en una monografía de investigación altamente especializada. 
